\title{
Retroactive revaluation of an odor-taste association
}

\author{
JUSTIN A. HARRIS and R. FREDERICK WESTBROOK \\ University of New South Wales, Sydney, New South Wales, Australia
}

\begin{abstract}
Rats showed an increase or decrease in preference for an aqueous odor conditioned stimulus (CS) presented in compound with a palatable (sucrose) or unpalatable (quinine) taste unconditioned stimulus (US), respectively. Four experiments then studied the consequences for odor preference of presenting water in the context where the odor-taste compound had previously occurred (i.e., no-CSno-US experience). Exposure to that context increased the preference for the odor associate of sucrose and decreased preference for the odor associate of quinine. The increased preference for the odor associate of sucrose was contingent on reexposure to the context where the odor-sucrose compound had occurred (i.e., retroactive revaluation) and was reduced by preexposure to the context where the odor-sucrose compound was to occur (i.e., latent inhibition of the context). The retroactive revaluation of within-event learning caused by exposures to the associated context is discussed in terms of (1) the modified version of the SOP model (Wagner, 1981) proposed by Dickinson and Burke (1996) and (2) the comparator model (Miller \& Matzel, 1988).
\end{abstract}

Contemporary theories of associative learning have been strongly influenced by demonstrations that responding to a target conditioned stimulus (CS) is modulated by the status of that and other CSs present during training. Interference effects such as overshadowing, blocking, and latent inhibition have inspired theories that describe the mechanisms by which CSs compete for attentional processing and for associative strength with the unconditioned stimulus (US) (Mackintosh, 1975; Pearce \& Hall, 1980; Rescorla \& Wagner, 1972; Wagner, 1981). Thus, these theories typically attribute variations in conditioned responding to differences in the acquisition of associations during CS-US pairings. Moreover, these theories assume that modulation of CS-US associations can occur only when the animal is directly exposed to the CS.

However, this view has been challenged by demonstrations that performance to a CS can be increased or decreased retroactively by changing the associative strength between other CSs and the US. For example, studies investigating learning of stimulus-outcome relations in human subjects have shown that performance to an overshadowed stimulus can be retroactively increased by posttraining exposures to the paired stimulus. Specifically, if subjects learn the association between a compound cue, $\mathrm{AB}$, and a particular outcome, then the predictive value accorded A can be retroactively decreased by paired presentations of B and the outcome (Chapman, 1991; Dickinson \& Burke, 1996; Shanks, 1985; Van Hamme \& Wasserman, 1994; Williams, Sagness, \& McPhee, 1994) or retro-

The research was supported by a grant from the Australian Research Council, and the experimental procedures were approved by the Animal Care and Ethics Committee (ACEC) of the University of New South Wales. The authors are grateful to P. Lovibond and R. Richardson for comments. Correspondence should be addressed to J. A. Harris, School of Psychology, University of New South Wales, Sydney, 2052 NSW, Australia (e-mail: j.harris@unsw.edu.au). actively increased by presentations of B in the absence of the associated outcome (Dickinson \& Burke, 1996; Van Hamme \& Wasserman, 1994).

Evidence for retroactive changes in CS-US associations in studies with nonhuman animal subjects has been more equivocal. On the one hand, these studies have failed to observe a decrease in conditioned performance to $\mathrm{A}$ when reinforced exposures to an $\mathrm{AB}$ compound were followed by reinforced exposures to B (e.g., Kalat \& Rozin, 1972; Schweitzer \& Green, 1982). On the other hand, there are numerous studies, using fear conditioning in rats, that have shown that overshadowing of one element $(A)$ of a reinforced $\mathrm{AB}$ compound can be reversed by posttraining extinction of B (Kaufman \& Bolles, 1981; Matzel, Schachtman, \& Miller, 1985; Yin, Grahame, \& Miller, 1993). Similarly, latent inhibition of a CS was attenuated by extensive exposure to the training context (i.e., context extinction) following CS-US pairings (Grahame, Barnet, Gunther, \& Miller, 1994). However, in several other studies in which rats were trained with an $\mathrm{AB}$ compound followed by extinction of $B$, decreased performance to A was observed (e.g., Holland \& Ross, 1981; Rescorla \& Cunningham, 1978; Speers, Gillan, \& Rescorla, 1980). For instance, Durlach and Rescorla (1980) reported that rats made ill after exposure to a taste-odor compound subsequently displayed a weaker aversion to that odor if the taste element of the poisoned compound was extinguished prior to test. These investigators argued that the rats had learned within-event associations between the odor and taste, which enabled the odor to suppress ingestive behavior because it activated the representation of the strongly averted taste. Thus, extinction of the taste had reduced test performance to the odor by reducing the amount of associative strength that the odor could borrow from the taste.

The general significance of these findings is that animals initially trained with an $\mathrm{AB}$ compound followed by 
reinforced or nonreinforced presentations of B may not readily show evidence for retroactive revaluation of $A$ because the status of B contributes directly to test performance (Durlach, 1989). In other words, it is possible that extinction of $\mathrm{B}$, for instance, retroactively increases the predictive value of $\mathrm{A}$ but that the resulting increase in performance to $\mathrm{A}$ is traded against a decrease in that performance caused by the inability of $A$ to borrow associative strength from B (Durlach \& Rescorla, 1980). Thus, the detection of evidence for retroactive revaluation may depend on whether $A$ borrows associative strength from $B$ on test. For example, in several of the experiments by Miller and his colleagues (e.g., Grahame et al., 1994; Yin et al., 1993), retroactive revaluation of a discrete CS was brought about by extinction of the training context. In these experiments, the critical CS-context associations would have been relatively weak in comparison with the strong odor-taste associations established in the experiment reported by Durlach and Rescorla (1980). Therefore, extinction of the context-US association would not undermine test performance to the CS, thereby allowing the retroactive increase in predictive value of the CS to be detected as increased test performance to that cue.

Demonstrations of retroactive changes in performance to a CS represent a major challenge to theories of associative learning (Durlach, 1989). However, these effects have been demonstrated only in a limited number of learning paradigms. Thus, it is important to determine the generality of these retroactive changes by investigating their occurrence in other learning paradigms. Accordingly, the present experiments investigated whether rats show retroactive revaluation of within-event associations between an odor and a taste. Rats were exposed to a solution composed of an odor and a hedonic taste in Context 1 in order to establish associations between the taste and the odor and between each of these cues and Context 1. Rats were then reexposed to Context 1 or to a second context without the odor or taste. An increase in performance to the odor on test among rats reexposed to Context 1 , but not among rats exposed to Context 2 , would constitute evidence for retroactive revaluation.

\section{EXPERIMENT 1}

The aim of Experiment 1 was to show that rats learn about the relation between the elements of a simultaneous compound composed of an aqueous odor (almond) and a taste (sucrose or quinine). Rats received presentations of an almond-sucrose compound (Group AS) or separate presentations of the almond and sucrose (Group A/S), whereas other rats were exposed to an almond-quinine compound (Group AQ) or separate presentations of the almond and the quinine (Group A/Q). All rats were tested for consumption of almond versus water to determine whether the preference for almond had been increased or decreased by its presentation in a compound with the sweet or bitter taste, respectively.

\section{Method}

Subjects. The subjects were experimentally naive male Wistar rats obtained from the colony of specific-pathogen-free rats maintained by the Combined Universities Laboratory Animal Services (Sydney, Australia). They weighed 200-300 g. The rats were housed in plastic boxes $(65 \times 40 \times 22 \mathrm{~cm})$ where pelleted food was continuously available. There were no more than 8 rats per box, and the boxes were kept in a colony room maintained on a natural light:dark cycle. The experiments were conducted between 9:00 a.m. and 3:00 p.m.

Apparatus. The apparatus consisted of 10 white plastic buckets ( $38 \times 20 \mathrm{~cm}$, height $\times$ diameter) located on a bench in one of the rooms in the laboratory. Two inverted calibrated cylinders spaced $6 \mathrm{~cm}$ apart were mounted to the outside of each bucket. The stainless steel, ball-bearing-tipped spouts of the cylinders protruded $2 \mathrm{~cm}$ into the bucket at a height of $3 \mathrm{~cm}$ above the floor.

Procedure. The rats were weighed and handled each day across 3 days and then were placed on a fluid-deprivation schedule, during which the rats were allowed $20 \mathrm{~min}$ of water access each day at 6:00 p.m. On Day 1, the rats were transported to the laboratory at 9:00 a.m. and placed in the plastic buckets for $10 \mathrm{~min}$. The rats in Group AS were exposed to a solution composed of $1 \% \mathrm{v} / \mathrm{v}$ almond essence (Aeroplane, Australia) and 3.4\% w/v sucrose (BDH Chemicals, Australia); the rats in Group AQ received a solution composed of the almond and $0.001 \% \mathrm{w} / \mathrm{v}$ quinine (BDH Chemicals, Australia). The rats in Groups $A / S$ and $A / Q$ were given water containing the almond alone (all $n s=10$ ). Six hours later, all rats were again transported to the laboratory and placed in the buckets for $10 \mathrm{~min}$. The rats in Groups AS and AQ then received water, whereas those in Groups A/S and A/Q received sucrose and quinine, respectively. On Day 2, this procedure was repeated except that the order of trials was reversed. Thus, the rats in Groups AS and AQ received water in the morning and the compound solution (either almondsucrose or almond-quinine) in the afternoon, whereas the rats in Groups $A / S$ and $A / Q$ were exposed to their respective tastes (sucrose and quinine) in the morning and to the aqueous odor (almond) in the afternoon. On the following morning, Day 3, all rats were tested. They were transported to the laboratory and placed in the plastic buckets for $10 \mathrm{~min}$. One cylinder contained $1 \%$ almond in water; the other cylinder contained water. After $5 \mathrm{~min}$, the position of each cylinder was reversed.

\section{Results and Discussion}

The mean consumption of almond and water on test by the rats in each of the four groups is shown in the upper portion of Figure 1. It is clear that the rats exposed to the almond-sucrose compound (Group AS) consumed more and those exposed to the almond-quinine solution (Group AQ) consumed less of the almond-scented water than did the control rats (Groups A/S and A/Q). Preference ratios consisting of almond consumption over total consumption for the rats in each of the groups are shown in the lower portion of Figure 1. These ratios were analyzed by means of a contrast testing procedure (Hays, 1972 ). With $\alpha$ set at .05 , the critical $F$ value was 4.11 . There were no significant differences between the ratios of the rats trained with sucrose or with quinine $[F(1,36)=2.92]$. However, the rats trained with the almond-sucrose compound showed a significantly stronger preference for the almond-scented water than did the rats that had received separate exposures to the almond and sucrose $[F(1,36)=$ $7.41]$. In contrast, the rats trained with the almond-quinine 

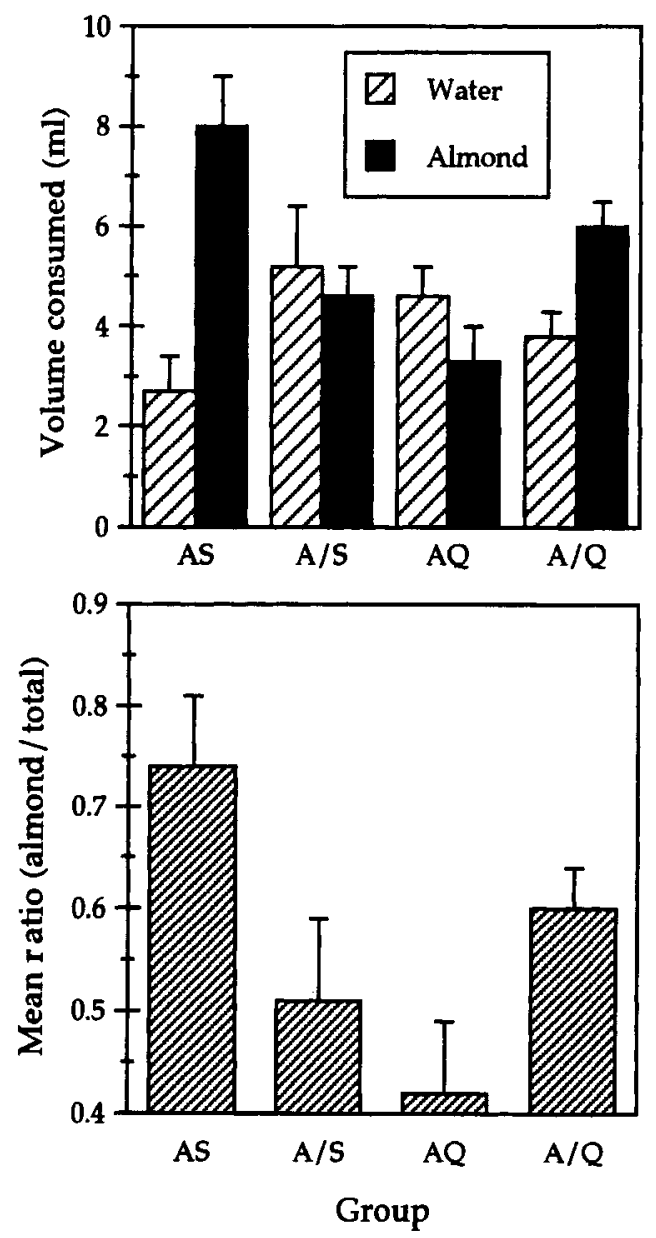

Figure 1. Test intake (mean and $S E M$ ) of almond and water (upper panel) and the mean ratios of almond intake over total intake (lower panel) for the rats in Experiment 1. The rats had been exposed to the almond solution in compound with sucrose (AS) or explicitly unpaired with sucrose (A/S), or in compound with quinine (AQ) or explicitly unpaired with quinine (A/Q).

compound showed a significantly weaker almond preference than did the rats that had received separate exposures to the almond and quinine $[F(1,36)=4.81]$. Thus, two exposures are sufficient for rats to learn about the relation between the odor and taste elements of a simultaneous compound. Moreover, the expression of this withinevent learning reflects the hedonic properties of the taste such that sucrose increased and quinine decreased the preference for its odor associate in the compound.

\section{EXPERIMENTS 2A AND 2B}

The aim of Experiments $2 \mathrm{~A}$ and $2 \mathrm{~B}$ was to study whether performance to the almond associate of sucrose or of quinine can be modified by exposure to the context in which the rats had received presentations of the almond-sucrose or almond-quinine compounds. In each experiment, rats received a single exposure to a solution composed of almond and sucrose (Experiment $2 \mathrm{~A}$ ) or of al- mond and quinine (Experiment 2B). The rats then received water either in the training chamber (reexposed groups) or in their boxes in the colony room (not-reexposed groups). A demonstration that reexposure to the training context provokes retroactive revaluation of the odor-taste association would involve showing that the rats reexposed to the training chamber display a greater preference for the almond associate of the sucrose (Experiment $2 \mathrm{~A}$ ) and a greater rejection of the almond associate of the quinine (Experiment $2 \mathrm{~B}$ ). In both experiments, rats were tested in a context that was different from the one in which they had received the compound to eliminate any direct contribution from that context to the test performance.

\section{Method}

Subjects and Apparatus. The subjects were experimentally naive male Wistar rats weighing $300-400 \mathrm{~g}$. They were obtained from the same source and maintained under the same conditions described for Experiment 1. There were 24 rats in each experiment. The rats were trained with the almond-sucrose and almond-quinine solutions used in Experiment 1. Two sets of contexts were used. The rats were trained in individual boxes $(30 \times 35 \times 20 \mathrm{~cm}$, depth $\times$ width $\times$ height). The ceiling and side walls of these boxes were constructed of wood painted white, and the opening front wall was made of Perspex. The floor was constructed of stainless steel. Two inverted calibrated cylinders spaced $6 \mathrm{~cm}$ apart were mounted to the outside of each box. The stainless steel, ball-bearing-tipped spouts of the cylinders protruded $2 \mathrm{~cm}$ into the bucket at a height of $3 \mathrm{~cm}$ above the floor. The second set of chambers where the rats were tested was made up of the white plastic buckets described previously. The wooden boxes and the plastic buckets were located in different rooms in the laboratory.

Procedure. The rats were handled and placed on the fluiddeprivation schedule described for Experiment 1 . On the morning of Day 1, the rats received 10-min access to an almond-sucrose solution (Experiment 2A) or an almond-quinine solution (Experiment $2 \mathrm{~B}$ ) in the wooden boxes. In each experiment, the rats were then allocated to two weight-matched groups $(n \mathrm{~s}=12)$. Six hours later, the reexposed rats were returned to the wooden boxes where they received water for $10 \mathrm{~min}$, whereas the not-reexposed rats were handled and given 10-min access to water in their home boxes in the colony room. At 6:00 p.m., all rats received a further 20-min access to water in their home boxes in the colony room. The following morning, all rats were given a 10-min choice test in the buckets in the manner described for Experiment 1.

\section{Results and Discussion}

The mean consumption of almond and water by the rats in each of the two groups in both experiments are shown in the upper portion of Figure 2. The left panel shows the results from Experiment 2A, and the right panel shows those from Experiment 2B. The lower portion of this figure shows the preference ratios for the rats in Experiment 2A (left panel) and Experiment 2B (right panel). Inspection of these panels suggests that reexposure to the context in the absence of the expected compound modified preferences for the almond associate of the sweet or bitter taste. The statistical analysis of the ratios obtained in Experiment $2 \mathrm{~A}$ confirmed that the reexposed rats showed a significantly greater preference for the almond associate of the sucrose than did the notreexposed rats $[t(22)=2.14, p<.05]$. In contrast, the re- 


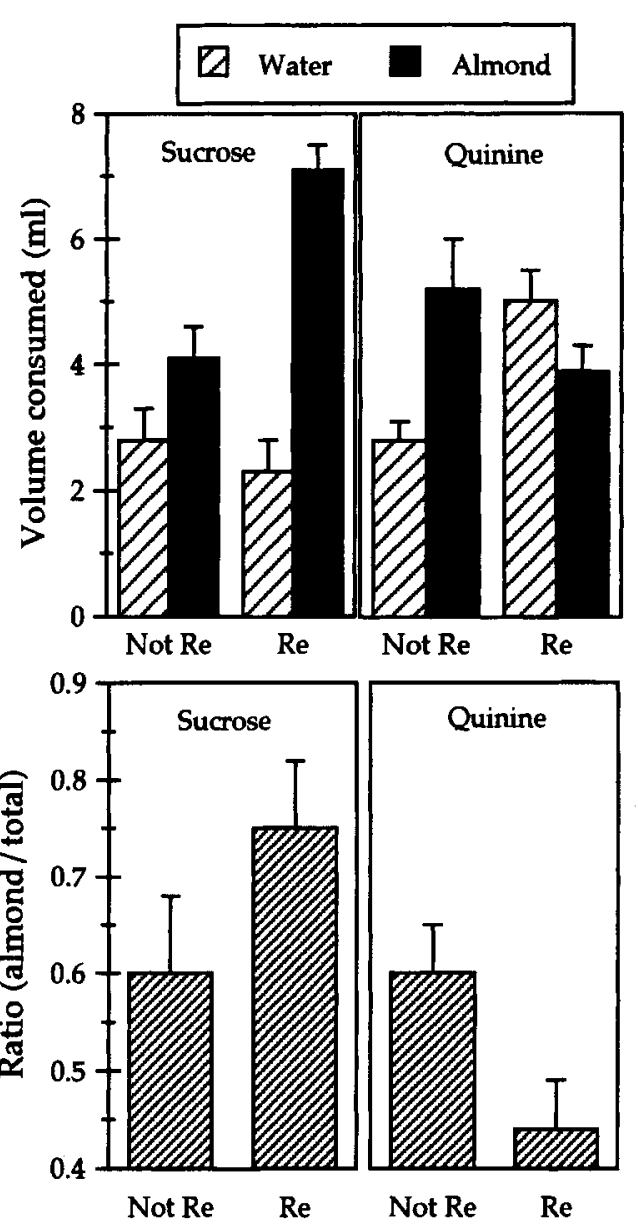

Figure 2. Test intake (mean and $S E M$ ) of almond and water (upper panel) and the mean ratios of almond intake over total intake (lower panel) for the rats in Experiment 2A (left) and Experiment $2 B$ (right). The rats had been exposed once to an almondsucrose solution or an almond-quinine solution and subsequently either were reexposed to the training chamber but given water (Re) or were given water in their home cages (Not Re).

exposed rats in Experiment 2B showed a significantly greater avoidance of the almond associate of the quinine than did the not-reexposed rats $[t(22)=2.66, p<.05]$.

It may be noted that the preference ratios for the notreexposed rats in each experiment were similar, though the rats in Experiment $2 \mathrm{~A}$ had received the almond in combination with sucrose and those in Experiment 2B had been presented with the almond in combination with quinine. This similarity suggests that a single exposure to almond in compound with either sucrose or quinine did not allow the rats to acquire an odor-taste association sufficiently strong to be detected in performance on the choice test. Nevertheless, it is clear that the preference resulting from that single exposure was modified in each experiment by reexposure to the context in the absence of the expected compound.

\section{EXPERIMENT 3}

The design used in Experiment 2A involved reexposing rats to the context in which the almond-sucrose compound had been presented. However, that reexposure constituted an additional context exposure for the reexposed rats relative to that for the not-reexposed rats that had remained in their home boxes in the colony room. This additional context exposure might have reduced the levels of neophobia provoked by the test presentation of almond in the novel context, with the consequence that the reexposed rats displayed a stronger almond preference than the not-reexposed rats because the former were less neophobic than the latter. The design of Experiment 2B was also subject to this confound, but the preference ratios obtained in this experiment were the opposite of those expected on the basis of differential neophobic reactions elicited by the test presentation of almond in the novel context. Therefore, the aim of Experiment 3 was to examine whether the results obtained in Experiment $2 \mathrm{~A}$ were due to the fact that the reexposed rats had received an additional exposure to a context and the not-reexposed rats had not. In Experiment 3, reexposed and not-reexposed rats were exposed to the almond-sucrose compound in Context 1 . Subsequently, reexposed rats received water in Context 1, whereas not-reexposed rats received water in Context 2. The rats in both groups were then tested for almond preference in Context 3.

\section{Method}

Subjects and Apparatus. The subjects were 40 experimentally naive male Wistar rats weighing $300-400 \mathrm{~g}$. They were obtained from the same source and maintained under the same conditions described for Experiment 1. Three sets of contexts were used. The first consisted of the white-painted boxes with metal floors described for Experiments $2 \mathrm{~A}$ and $2 \mathrm{~B}$. The second set of contexts consisted of unpainted wooden boxes $(29 \times 29 \times 29 \mathrm{~cm})$ with wiremesh (spaced $1 \times 2.5 \mathrm{~cm}$ ) floors. Two inverted calibrated cylinders spaced $6 \mathrm{~cm}$ apart were mounted to the outside of each box. The stainless steel, ball-bearing-tipped spouts of the cylinders protruded $2 \mathrm{~cm}$ into the bucket at a height of $3 \mathrm{~cm}$ above the floor. The third test context consisted of the plastic buckets. Each of the contexts was located in separate rooms in the laboratory.

Procedure. The rats were handled and subjected to the fluiddeprivation schedule described for Experiment 1. On the morning of Day 1 , the rats received 10 -min access to the almond-sucrose solution in Context 1. For half of the rats, Context 1 consisted of the boxes with the stainless steel floors; for the remainder of the rats, this context was supplied by the boxes with the wire-mesh floors. Six hours later, the reexposed rats received a further 10 -min exposure to Context 1 in which water was available. This context was either the boxes with the stainless steel floors or the boxes with the wire-mesh floors. At this time, the not-reexposed rats received 10 -min access to water in Context 2 . For the not-reexposed rats initially exposed to the boxes with the stainless steel floors, Context 2 consisted of the boxes with the wire-mesh floor; for the notreexposed rats initially exposed to the boxes with the wire-mesh floors, Context 2 consisted of the boxes with the stainless steel floors (all $n \mathrm{~s}=10$ ). The following morning, all rats were tested for consumption of almond-scented water versus water. The test lasted 
for $10 \mathrm{~min}$, and the position of the cylinders was reversed after $5 \mathrm{~min}$. The test took place in the plastic buckets.

\section{Results and Discussion}

The two contexts used during training did not differentially affect test performance. Therefore, the preference ratios were collapsed across the contexts employed. The mean consumption of almond and water by the rats in each of the two groups are shown in the upper portion of Figure 3; the corresponding preference ratios are shown in the lower portion of this figure. The statistical analysis confirmed that the rats exposed to water in the context in which the compound had been presented showed significantly greater almond preferences than did the rats exposed to water in a different context $[t(38)=2.31, p<$ $.05]$. This shows that retroactive revaluation of an almond-sucrose association, like that previously observed for the almond-quinine association (Experiment 2B), is contingent on rats being exposed to a context in which they had come to expect the almond-sucrose compound, and not on differential neophobia to the test context.

\section{EXPERIMENT 4}

The aim of Experiment 4 was to determine whether reexposure to a context in which an odor-sucrose compound had been presented causes a retroactive revaluation that is specific to that odor. To accomplish this aim, the experiment used a $2 \times 2$ factorial design in which all rats were exposed to an almond-sucrose compound in one context (Context ALM) and to a vanilla-sucrose compound in a second context (Context VAN). The rats were then reexposed to either Context ALM or Context VAN (Factor 1) in which they received water. Finally, the rats were tested in a third context with either almond versus water or vanilla versus water (Factor 2 ). If retroactive

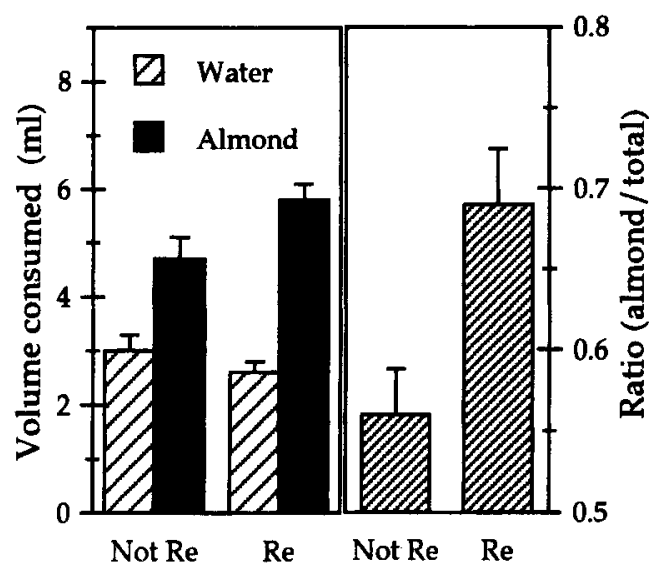

Figure 3. Test intake (mean and $S E M$ ) of almond and water (left panel) and the mean ratios of almond intake over total intake (right panel) for the rats in Experiment 3. The rats had been exposed once to an almond-sucrose solution and later either were reexposed to the training chamber $(\mathrm{Re})$ or were exposed to a different chamber (Not Re). revaluation is stimulus specific, rats reexposed to Context ALM will show an increased preference for almond but not vanilla, whereas rats reexposed to Context VAN will show an increased preference for vanilla but not almond. Such an effect would be identified by a significant interaction between the reexposure context (Factor 1) and the test odor (Factor 2).

\section{Method}

Subjects and Apparatus. The subjects were 24 experimentally naive male Wistar rats weighing $300-400 \mathrm{~g}$. They were obtained from the same source and maintained under the same conditions described for Experiment 1. The three contexts described in Experiment 3 were used here, with the plastic buckets again serving as the test context.

Procedure. The rats were handled and placed on the fluid deprivation schedule described previously. On Day 1 , rats received a target fluid on two occasions, in the morning and $6 \mathrm{~h}$ later in the afternoon. One target was the almond-sucrose compound, while the second was a compound composed of sucrose and $2 \%$ natural vanilla essence (Queen, Sydney). The almond-sucrose solution was presented in one context (Context ALM) and the vanilla-sucrose compound was presented in a second context (Context VAN). For half of the rats, the almond-sucrose compound was presented in the boxes with the stainless steel floors and the vanilla-sucrose compound was presented in the boxes with the wire-mesh floors. For the remainder, the almond-sucrose compound was presented in the boxes with the wire-mesh floors and the vanilla-sucrose compound was presented in the boxes with the stainless steel floors. The order in which rats were exposed to the compounds was counterbalanced within each of these conditions. On Day 2, rats received a 10-min exposure to water in either Context ALM or VAN. On Day 3, half of the rats reexposed to Context ALM and half of those reexposed to Context VAN were tested with almond versus water, while the remainder were tested with vanilla versus water. The test took place in the plastic buckets and was conducted in the manner described previously.

\section{Result and Discussion}

The mean intakes on test of almond and water are shown in the upper left panel of Figure 4; the mean intakes of vanilla and water are shown in the upper right panel. The preference ratios based on these intakes are shown in the lower panels of this figure. An inspection of the left panel of the upper portion shows that the rats reexposed to Context ALM drank more of the almond than did the rats reexposed to Context VAN. In contrast, the right panel shows that the rats reexposed to Context VAN drank more vanilla than did the rats reexposed to Context ALM. The statistical analysis of the ratios confirmed these observations. With $\alpha$ set at .05, the critical $F$ value was 4.33 . There were no significant main effects between the ratios of the rats tested with almond or vanilla $[F(1,21)=1.5]$ or between the ratios of the rats reexposed to Context ALM or Context VAN $[F(1,21)<$ $1.0]$. However, there was a statistically significant interaction between these factors $[F(1,21)=11.6]$, which shows that reexposure to a context in which the rats had drunk an odor-sucrose compound caused a retroactive revaluation of the odor-sucrose association that was specific to that odor. 

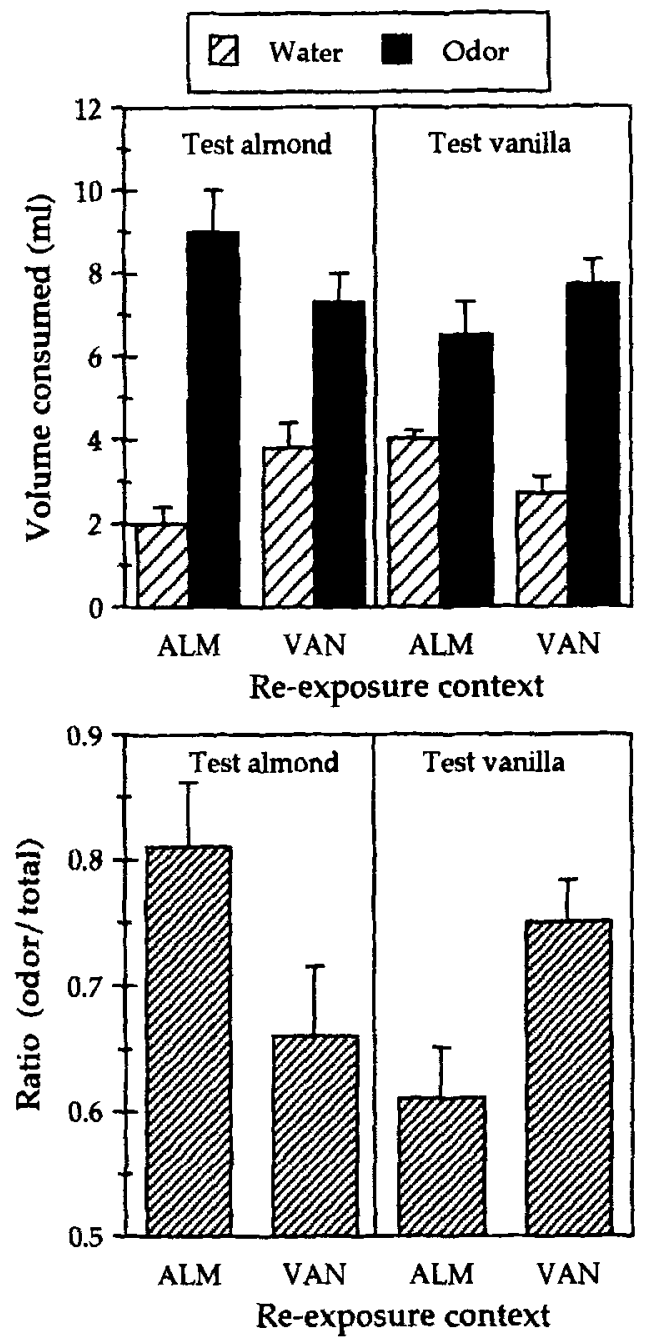

Figure 4. Test intake (mean and $S E M$ ) of almond and water (top left panel) or vanilla and water (top right panel) and the mean ratios of almond intake over total intake (bottom left panel) or of vanilla intake over total intake (bottom right panel) for the rats in Experiment 4. The rats had been exposed once to an almond-sucrose compound solution in one chamber (Context ALM) and exposed once to a vanilla-sucrose compound solution in a different chamber (Context VAN). Following this, the rats had been reexposed to either Context ALM or Context VAN but were given water.

\section{EXPERIMENT 5}

There is evidence that rats fail to learn associations between a very familiar context and events experienced in that context. For example, rats showed little fear of a chamber in which they were shocked if they had been extensively exposed to the chamber beforehand (Kiernan $\&$ Westbrook, 1993). In addition to producing deficits in learning or performance of context-US associations, extensive preexposure to a context produces similar deficits in learning or performance of the relation between that context and a CS. For instance, latent inhibition of a
CS is normally specific to the context in which the CS was presented, but is context independent when the CS is presented in an already-familiar context (McLaren, Bennett, Plaisted, Aitken, \& Mackintosh, 1994). These results suggest that a preexposed context fails to enter into an association with a CS (e.g., McLaren, Kaye, \& Mackintosh, 1989) or to act as a retrieval cue for what has been learned about that CS (e.g., Bouton, 1993). However, regardless of their interpretation, these results suggest that exposure to a context in the absence of an expected almond-sucrose compound will be less effective in causing retroactive revaluation when that context had been preexposed than when the compound had been presented in a novel context.

The aim of Experiment 5 was to examine the effect of preexposure to a context on the retroactive revaluation produced by reexposure to that context. The experiment employed a $2 \times 2$ factorial design, with one factor being preexposure to the training chamber versus a different chamber, and the other factor being reexposure to the training chamber versus a different chamber. In Stage 1, all rats were exposed to water either in the training context (preexposed groups) or in a different context (notpreexposed groups). In Stage 2, all rats were exposed to the almond-sucrose compound in the training context. In Stage 3, they were exposed to water either in the training context (reexposed groups) or in a different context (not-reexposed groups). The question of interest is whether preexposure to the training chamber reduces the retroactive revaluation of the almond-sucrose association caused by a reexposure to that chamber.

\section{Method}

Subjects and Apparatus. The subjects were 44 experimentally naive male Wistar rats weighing $300-400 \mathrm{~g}$. They were obtained from the same source and maintained under the same conditions described for Experiment 1 . In addition to the three sets of contexts used in Experiment 3, a fourth context consisted of rack-mounted cages $(24 \times 15 \times 19 \mathrm{~cm}$, depth $\times$ width $\times$ height $)$ constructed of 2 -mm-thick steel bars spaced $1 \times 5 \mathrm{~cm}$. Two inverted calibrated cylinders were mounted against the external wall of each cage. The stainless steel, ball-bearing-tipped spouts of the cylinders protruded $2 \mathrm{~cm}$ into each cage at a height of $3 \mathrm{~cm}$ above the floor. These cages were located in the animal colony room, whereas the other three contexts, including the plastic buckets that served as the test context, were located in separate rooms in the laboratory.

Procedure. The rats were handled and placed on the fluiddeprivation schedule described for Experiment 1. On Days 1-4, the rats in Groups Preexposed/Reexposed and Preexposed/NotReexposed received $10-\mathrm{min}$ exposures to water in the training chambers each morning and afternoon. For half of the rats in each of these groups, these training chambers were the boxes with the stainless steel floors; for the remainder, they were the boxes with the wire-mesh floors. On these days, the rats in Groups Not-Preexposed/ Reexposed and Not-Preexposed/Not-Reexposed received 10-min exposures to water each morning and afternoon in the rack-mounted cages located in the colony room (all $n s=11$ ). On the morning of Day 5, all rats were transported to the laboratory and exposed for 10 min to the training context (the boxes with stainless steel floors or those with wire-mesh floors) and presented with the almond-sucrose compound. To avoid differences between the intakes of the almond-sucrose compound by preexposed and not-preexposed 
rats, the intakes of the latter rats were recorded. The amount drunk by a given rat in the not-preexposed groups was then provided to a randomly selected rat in the preexposed groups. In the afternoon of Day 5, the rats in Group Preexposed/Reexposed and the rats in Group Not-Preexposed/Reexposed were exposed for $10 \mathrm{~min}$ to water in the context in which they had received the almond-sucrose compound. For half of the rats in each of these groups, this context was the boxes with the stainless steel floors; for the remainder, it was the boxes with the wire-mesh floors. Also in the afternoon of Day 5 , the rats in Groups Preexposed/Not-Reexposed and Not-Preexposed/ Not-Reexposed received water in the other context: for half of the rats, it was the boxes with the stainless steel floors; for the remainder, it was the boxes with the wire-mesh floor. On the morning of Day 6, all rats were tested for consumption of almond versus water.
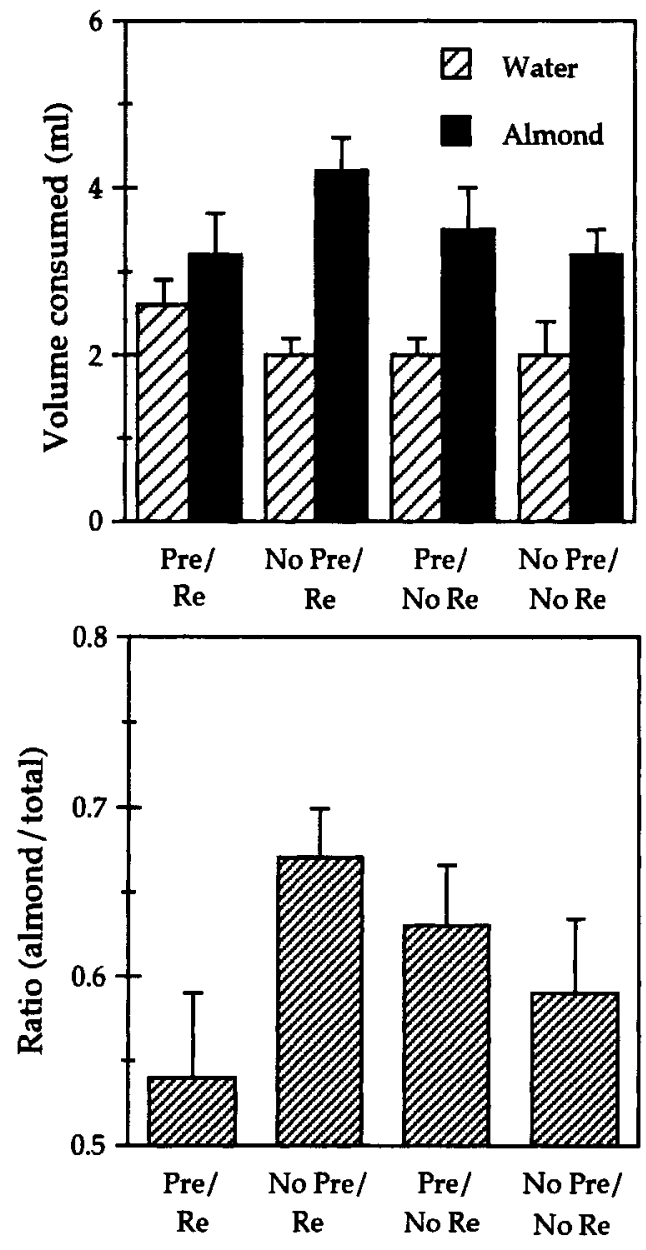

Figure 5. Test intake (mean and $S E M$ ) of almond and water (upper panel) and the mean ratios of almond intake over total intake (lower panel) for the rats in Experiment 5 . The rats had been preexposed to the training chamber and reexposed to that chamber (Pre/Re), preexposed to a different chamber but reexposed to the training chamber (No Pre/Re), preexposed to the training chamber but reexposed to a different chamber (Pre/No Re), or preexposed and reexposed to a different chamber (No Pre/No Re). Between preexposure and reexposure, all rats were given an almond-sucrose solution in the training chamber.
The test took place in the plastic buckets and was conducted in the manner described previously.

\section{Results and Discussion}

The mean consumption of almond and water for the rats in each of the four groups are shown in the upper portion of Figure 5; the ratios based on these intakes are shown in the lower portion of this figure. It is clear that the not-preexposed/reexposed rats showed the greatest preference for almond, whereas the preexposed/reexposed rats showed the lowest preference. There was little difference between the preexposed/not-reexposed rats and the not-preexposed/not-reexposed rats. With $\alpha$ set at .05 , the critical $F$ value was 4.09 for the statistical analysis conducted on the ratio scores. There were no significant main effects for preexposure or reexposure $[F \mathrm{~s}(1,40) \leq 1.81]$. However, there was a significant interaction between these factors $[F(1,40)=4.63]$, confirming that preexposure had interfered with the effect of reexposure. Simple effect contrasts identified that the source of this interaction was a difference between the preexposed/reexposed rats and the not-preexposed/reexposed rats $(F=4.28)$. There was no significant difference between the preexposed/not-reexposed rats and the not-preexposed/notreexposed rats $(F<1.0)$. Thus, preexposure to the training chamber prevented the increase in almond preference otherwise seen in the rats reexposed to the training chamber before test.

According to the ratio data, the rats in Group Preexposed/Reexposed showed the lowest preference for almond, suggesting that these rats were impaired in learning or performance of the almond-sucrose association. Inspection of the upper panel of Figure 5 indicates that this reduced preference was largely due to an increased intake of water among these rats. The reason for this increase in water intake is unclear. Nevertheless, equivalent volumes of almond were consumed by the rats in Group Preexposed/ Reexposed and by those in the two not-reexposed groups, and these volumes were less than that consumed by the rats in Group Not-Preexposed/Reexposed.

\section{GENERAL DISCUSSION}

Our series of experiments has confirmed that rats learn about the relation between an odor CS and a hedonic taste US presented in a simultaneous compound. They exhibited this learning in a preference for the CS when its associated US was sucrose and a rejection when the associated US had been quinine. These preferences were changed by postcompound exposures to the training context. Specifically, the rats exposed to the context in the absence of the expected CS-US compound (i.e., context extinction) subsequently showed an increased preference for the CS when the associated US had been sucrose and an increased rejection of the CS when its US had been quinine. Thus, our experiments have confirmed that ex- 
tinction of the training context results in retroactive revaluation of a within-compound CS-US association, just as that extinction has provoked retroactive revaluation of a between-event CS-US association (e.g., Grahame et al., 1994; Yin et al., 1993).

Two distinct theoretical analyses have been offered to explain demonstrations of retroactive revaluation. According to one analysis, best characterized by the "comparator" theory of Miller and his colleagues (e.g., Kasprow, Schachtman, \& Miller, 1987; Miller \& Matzel, 1988), performance to a CS is not determined by the strength of the association between the CS and the US but rather by the predictive value of the CS relative to other signals for the US. For example, exposed to pairings of a CS and a US in a particular context, rats form not only CS-US associations but also context-US and context$\mathrm{CS}$ associations. Performance to the CS is in part determined by the status of the context-US association. Therefore, extinction of that association (by exposure to the context in the absence of the US) establishes the CS as the better predictor of the US and thereby increases conditioned performance to the CS. With regard to the present experiments, when rats are reexposed to the context but given only water, this reexposure would extinguish the context-sucrose association, thereby enhancing performance to the odor.

The second theoretical analysis offered to explain retroactive revaluation is derived from the models of associative learning proposed by Rescorla and Wagner (1972) and Wagner (1981). The variable reinforcement rule proposed by Rescorla and Wagner holds that the change in associative strength of a $\mathrm{CS}, \Delta V$, that results from its pairing with a US is given by $\Delta V=\alpha \beta(\lambda-\Sigma V)$, where $\alpha$ and $\beta$ are learning rate parameters associated with the CS and the US, respectively, $\Sigma V$ is the sum of the associative strengths of all the CSs present on a learning occasion, and $\lambda$ (which has a positive value on occasions when the US occurs and a zero value on occasions when the US does not occur) is the value of $\sum V$ that completely predicts the US. Van Hamme and Wasserman (1994) have proposed a revision to this formal associative rule to explain retroactive revaluation effects such as observed here. These authors suggested that the $\alpha$ for a cue has a negative value on learning trials when that cue is absent. Thus, when rats are reexposed to the training context but given water, the $\left(\lambda-\sum V\right)$ term is negative because the taste US is absent, and the $\alpha$ value for the odor is also negative. Therefore, on this trial, $\Delta V$ for the odor is positive, and the strength of the odor-taste association increases.

Wagner's (1981) "sometimes opponent process" (SOP) model proposes that the nodes representing a stimulus are either inactive (I) or in two states of activation (termed A1 and A2). The presentation of that stimulus excites some proportion of its nodes from I to an Al state of activation, from which state they decay back to I through an A2 state. Excitatory associations are formed between the nodes of stimuli that are simultaneously activated into an $\mathrm{A} 1$ state, with the consequence that presentation of one excites the nodes of the other to an A2 state. The modification proposed by Dickinson and Burke (1996) that enables SOP to explain retroactive revaluation is that excitatory associations are formed not only between nodes simultaneously activated to an Al state but also when nodes are simultaneously active in an $\mathrm{A} 2$ state. Thus, in the case in which extinction training of $B$ retroactively increases the signal value of $\mathrm{A}$ following previous training in which the $\mathrm{AB}$ compound was reinforced, presentation of B simultaneously excites the nodes of its associates, $A$ and the US, into the A2 state of activation, with the result that the excitatory association between $A$ and the US is strengthened. In the present experiments, reexposing the rats to the context where they had previously drunk an odor-taste compound activated nodes corresponding to the odor and the taste into an A2 state, thereby strengthening the associative link between them.

The revised application of the Rescorla-Wagner rule asserts that the $\alpha$ for a cue is negative on learning trials when that cue is absent, but it fails to specify the processes that determine which absent cue has a negative $\alpha$ value. For instance, in Experiment 4, the rats were exposed to an almond-sucrose compound in one context (ALM) and to a vanilla-sucrose compound in a second context (VAN). Subsequently, the rats were exposed to one or other of these contexts in the absence of the compounds. Thus, the $\alpha$ values of both the almond and the vanilla should have been negative in the rats reexposed to either context, since each of these cues was absent on that reexposure. Hence, reexposure to either context should have retroactively revalued both the almond and the vanilla. However, retroactive revaluation was specific to the context associated with a particular odor-sucrose compound: The rats reexposed to Context ALM showed revaluation of the almond but not the vanilla, whereas the rats reexposed to Context VAN revalued the vanilla but not the almond. Therefore, the $\alpha$ value for almond, but not for vanilla, was negative only when the rats were reexposed to the context in which the expected almondsucrose was omitted, and the $\alpha$ value for the vanilla, but not for almond, was negative only when the rats were reexposed to the context in which the expected vanillasucrose compound was omitted.

By emphasizing the role of the association between $\mathrm{CSs}$ in determining retroactive revaluation, both the comparator model and the modified version of SOP proposed by Dickinson and Burke (1996) can account for the stimulus specificity observed in Experiment 4 . According to the comparator model, associations between the context and the odor determine which context enters into comparison with the odor on test (Miller \& Matzel, 1988). That is, test performance to almond was influenced by the status of the association between Context ALM and sucrose, whereas test performance to vanilla was influenced by the association between Context VAN and sucrose. Therefore, extinguishing the Context ALM-sucrose as- 
sociation increased performance to almond but not vanilla, and extinction of the Context VAN-sucrose association increased performance to vanilla but not almond. According to the revised version of SOP proposed by Dickinson and Burke, associations between the context and the odor determine which odor is revalued during context reexposure. Specifically, reexposure to Context ALM should have activated nodes corresponding to almond and sucrose into A2, thereby strengthening the almond-sucrose association but not the vanilla-sucrose association; reexposure to Context VAN should have activated nodes representing vanilla and sucrose into $A 2$, strengthening the vanilla-sucrose association but not the almond-sucrose association.

The comparator model and the revised version of SOP differ in their treatment of the results of Experiment 5. According to the comparator account, performance to the odor should have been affected not only by posttraining extinction of the context-sucrose association but also by preexposure to the training chamber, since either of these manipulations reduce the signal value of the context relative to that of the odor. Moreover, the signal value of the context should be reduced even further when rats are both preexposed and reexposed to the target context. Consistent with the predicted effect of preexposure, the rats preexposed to the training chamber showed a slight, but nonsignificant, increase in preference for almond in Experiment 5. However, in contrast to the effects expected from the combination of preexposure and reexposure, the rats in Experiment 5 that were preexposed to the target context and then subjected to reexposure showed a weaker odor preference than did the rats reexposed only to the training chamber. The weaker odor preferences exhibited by the rats that were both preeexposed and reexposed to the target context is consistent with the revised version of SOP, since the reduced associability of the preexposed context with the odor-sucrose compound would have undermined its ability on reexposure to strengthen the odor-sucrose association. However, it must be noted that this argument rests on the relatively weak evidence obtained here for a latent inhibitory effect of context preexposure: To be sure, prexposure did result in a slight increase in preference for the odor associate of the sucrose, but this increase was not statistically significant.

A revised version of SOP different from that put forward by Dickinson and Burke (1996) has been proposed by Hall (1996). In this version, excitatory learning between a CS and a US can proceed when the CS nodes are in A2 while the US nodes are in A1. This revision was proposed to explain demonstrations of "mediated conditioning." For instance, rats made sick after presentations of a tone that had previously signaled a food US subsequently exhibited an aversion to that food (Holland, 1981). To explain this finding, Holland $(1981,1990)$ argued that the signal had excited a memory of the food that was directly associated with illness. Hall (1996) casts this explanation in terms of SOP, arguing that the signal had activated nodes representing the food into an A2 state, and these nodes then became associatively linked to nodes representing the illness that were active in Al. This version of SOP differs from that proposed by Dickinson and Burke in two important respects. First, when event representations corresponding to a US and a CS are simultaneously activated into A1 and A2 states, respectively, excitatory associations are formed according to Hall, whereas inhibitory associations are established between these events according to Dickinson and Burke. Thus, the former but not the latter version of SOP can explain instances of mediated conditioning. Second, the DickinsonBurke revision of SOP allows for excitatory learning to take place between representations that are simultaneously excited into an A2 state of activation. Thus, the Dickinson-Burke version of SOP can account for retroactive revaluation effects such as reported in the present paper, whereas the Hall version cannot.

\section{REFERENCES}

Bouton, M. E. (1993). Context, time, and memory retrieval in the interference paradigms of Pavlovian learning. Psychological Bulletin, 114, 80-99.

ChapMan, G. B. (1991). Trial order affects cue interaction in contingency judgment. Journal of Experimental Psychology: Learning, Memory, \& Cognition, 17, 837-854.

Dickinson, A., \& Burke, J. (1996). Within-compound associations mediate the retrospective revaluation of causality judgements. Quarterly Journal of Experimental Psychology, 49B, 60-80.

Durlach, P. J. (1989). Learning and performance in Pavlovian conditioning: Are failures of contiguity failures of learning or performance? In S. B. Klein \& R. R. Mowrer (Eds.), Contemporary learning theories: Pavlovian conditioning and the status of traditional learning theory (pp. 19-59). Hillsdale, NJ: Erlbaum.

Durlach, P. J., \& Rescorla, R. A. (1980). Potentiation rather than overshadowing in flavor-aversion learning: An analysis in terms of within-compound associations. Journal of Experimental Psychology: Animal Behavior Processes, 6, 175-187.

Grahame, N. J., Barnet, R. C., Gunther, L. M., \& Miller, R. R. (1994). Latent inhibition as a performance deficit resulting from CScontext associations. Animal Learning \& Behavior, 22, 395-408.

HALL, G. (1996). Learning about associatively activated stimulus representations: Implications for acquired equivalence and perceptual learning. Animal Learning \& Behavior, 24, 233-255.

HAYs, W. L. (1972). Statistics for the social sciences. New York: Holt, Rinehart \& Winston.

Holland, P. C. (1981). Acquisition of representation-mediated conditioned food aversions. Learning \& Motivation, 12, 1-18.

Holland, P. C. (1990). Event representation of Pavlovian conditioning: Image and action. Cognition, 37, 105-131.

Holland, P. C., \& Ross, R. T. (1981). Within-compound associations in serial compound conditioning. Journal of Experimental Psychology: Animal Behavior Processes, 7, 228-24l

KalAT, J. W., \& Rozin, P. (1972). You can lead a rat to poison but you can't make him think. In M. E. P. Seligman \& J. L. Hager (Eds.), Biological boundaries of learning (pp. 115-122). New York: AppletonCentury-Crofts

Kasprow, W. J., Schachtman, T. R., \& Miller, R. R. (1987). The comparator hypothesis of conditioned response generation: Manifest conditioned excitation and inhibition as a function of relative excitatory associative strengths of $\mathrm{CS}$ and conditioning context at the time of testing. Journal of Experimental Psychology: Animal Behavior Processes, 13, 65-72.

Kaufman, M. A., \& Bolles, R. C. (1981). A nonassociative aspect of overshadowing. Bulletin of the Psychonomic Society, 18, 318-320.

Kiernan, M. J., \& Westbrook, R. F. (1993). Effects of exposure to a to-be-shocked environment upon the rat's freezing response: Evidence 
for facilitation, latent inhibition, and perceptual learning. Quarterly Journal of Experimental Psychology, 46B, 271-288.

Mackintosh, N. J. (1975). A theory of attention: Variations in the associability of stimuli with reinforcement. Psychological Review, 82, 276-298

Matzel, L. D., Schachtman, T. R., \& Miller, R. R. (1985). Recovery of an overshadowed association achieved by extinction of the overshadowing stimulus. Learning \& Motivation, 16, 398-412.

McLaren, I. P. L., Bennett, C., Plaisted, K., Aitken, M., \& MackINTOSH, N. J. (1994). Latent inhibition, context specificity, and context familiarity. Quarterly Journal of Experimental Psychology, 47B, $387-400$.

McLaren, I. P. L., Kaye, H., \& Mackintosh, N. J. (1989). An associative theory of the representation of stimuli: Applications to perceptual learning and latent inhibition. In R. G. M. Morris (Ed.), Parallel distributed processing: Implications for psychology and neurobiology (pp. 102-130). Oxford: Oxford University Press.

Miller, R. R., \& MATZEL, L. D. (1988). The comparator hypothesis: A response rule for the expression of associations. In G. H. Bower (Ed.), The psychology of learning and motivation (Vol. 22, pp. 51-92). San Diego: Academic Press.

Pearce, J. M., \& Hall, G. (1980). A model of Pavlovian learning: Variations in the effectiveness of conditioned but not of unconditioned stimuli. Psychological Review, 87, 532-552.

Rescorla, R. A., \& Cunningham, C. L. (1978). Within-compound flavor associations. Journal of Experimental Psychology: Animal Behavior Processes, 4, 267-275.

Rescorla, R. A., \& WAGNER, A. R. (1972). A theory of Pavlovian conditioning: Variations in the effectiveness of reinforcement and non- reinforcement. In A. H. Black \& W. F. Prokasy (Eds.), Classical conditioning II: Current research and theory (pp. 64-99). New York: Appleton-Century-Crofts.

SCHWEITZER, L., \& GREEN, L. (1982). Reevaluation of things past: A test of the retrospective hypothesis using a CER procedure with rats. Pavlovian Journal of Biological Science, 17, 62-68.

SHANKS, D. R. (1985). Forward and backward blocking in human contingency judgements. Quarterly Journal of Experimental Psychology, 37B, $1-21$.

Speers, M. J., Gillan, D. J., \& Rescorla, R. A. (1980). Withincompound associations in a variety of compound conditioning procedures. Learning \& Motivation, 11, 135-149.

Van Hamme, L. J., \& Wasserman, E. W. (1994). Cue competition in causality judgments: The role of nonpresentation of compound stimulus elements. Learning \& Motivation, 25, 127-151.

WAGNER, A. R. (1981). SOP: A model of automatic memory processing in animal behavior. In N. E. Spear \& R. R. Miller (Eds.), Information processing in animals: Memory mechanisms (pp. 5-47). Hillsdale, NJ: Erlbaum.

Williams, D. A., Sagness, K. E., \& McPhee, J. E. (1994). Configural and elemental strategies in predictive learning. Journal of Experimental Psychology: Learning, Memory, \& Cognition, 19, 174-188.

Yin, H., Grahame, N. J., \& Miller, R. R. (1993). Extinction of comparator stimuli during and after acquisition: Differential facilitative effects on Pavlovian responding. Learning \& Motivation, 24, 129-241.

(Manuscript received August 28, 1997; revision accepted for publication March 5, 1998.) 